\title{
Commissioning and early experiments of the PHELIX facility
}

\author{
V. Bagnoud $\cdot$ B. Aurand $\cdot$ A. Blazevic $\cdot$ S. Borneis $\cdot$ C. Bruske $\cdot$ B. Ecker $\cdot$ \\ U. Eisenbarth · J. Fils • A. Frank • E. Gaul • S. Goette · C. Haefner · T. Hahn · \\ K. Harres · H.-M. Heuck • D. Hochhaus • D.H.H. Hoffmann • D. Javorková • \\ H.-J. Kluge · T. Kuehl • S. Kunzer • M. Kreutz • T. Merz-Mantwill • P. Neumayer • \\ E. Onkels • D. Reemts • O. Rosmej • M. Roth • T. Stoehlker • A. Tauschwitz • \\ B. Zielbauer $\cdot$ D. Zimmer $\cdot$ K. Witte
}

Received: 12 October 2009 / Published online: 17 December 2009

(C) The Author(s) 2009. This article is published with open access at Springerlink.com

\begin{abstract}
At the Helmholtz center GSI, PHELIX (Petawatt High Energy Laser for heavy Ion eXperiments) has been commissioned for operation in stand-alone mode and, in combination with ions accelerated up to an energy of $13 \mathrm{MeV} / \mathrm{u}$ by the heavy ion accelerator UNILAC. The combination of PHELIX with the heavy-ion beams available at GSI enables a large variety of unique experiments. Novel research opportunities are spanning from the study of ionmatter interaction, through challenging new experiments in
\end{abstract}

V. Bagnoud $(\bowtie) \cdot$ A. Blazevic $\cdot$ S. Borneis $\cdot$ C. Bruske $\cdot$

B. Ecker · U. Eisenbarth · J. Fils · S. Goette · T. Hahn .

H.-J. Kluge · T. Kuehl · S. Kunzer · M. Kreutz

T. Merz-Mantwill $\cdot$ E. Onkels $\cdot$ D. Reemts $\cdot$ O. Rosmej .

T. Stoehlker - A. Tauschwitz - B. Zielbauer - D. Zimmer - K. Witte

GSI Helmholtzzentrum für Schwerionenforschung $\mathrm{GmbH}$,

Planckstr. 1, 64291 Darmstadt, Germany

e-mail: v.bagnoud@gsi.de

V. Bagnoud · A. Blazevic · B. Ecker · J. Fils · T. Stoehlker ·

B. Zielbauer

Helmholtz Institut Jena, Max Wien Platz 1, 07745 Jena, Germany

B. Aurand $\cdot$ D. Hochhaus $\cdot$ P. Neumayer

ExtreMe Matter Institute EMMI, GSI Helmholtzzentrum für

Schwerionenforschung GmbH, Planckstr. 1, 64291 Darmstadt,

Germany

B. Aurand · B. Ecker · T. Kuehl · D. Zimmer

Institut für Physik, Johannes Gutenberg-Universität Mainz,

Staudingerweg 7, 55128 Mainz, Germany

A. Frank · K. Harres · D.H.H. Hoffmann · M. Roth Institut für Kernphysik, Technische Universität Darmstadt, 64289 Darmstadt, Germany

E. Gaul

University of Texas at Austin, Austin, TX 78712, USA atomic physics, nuclear physics, and astrophysics, into the field of relativistic plasma physics.

\section{Introduction}

In the recent years, the commissioning and operation of high-energy petawatt lasers' (HEPW) facilities [1-3] around the world has enabled tremendous developments in the field of high-energy-density physics (HEDP) [4]. Motivated primarily by the needs for high-brilliance $\mathrm{X}$-ray sources used in

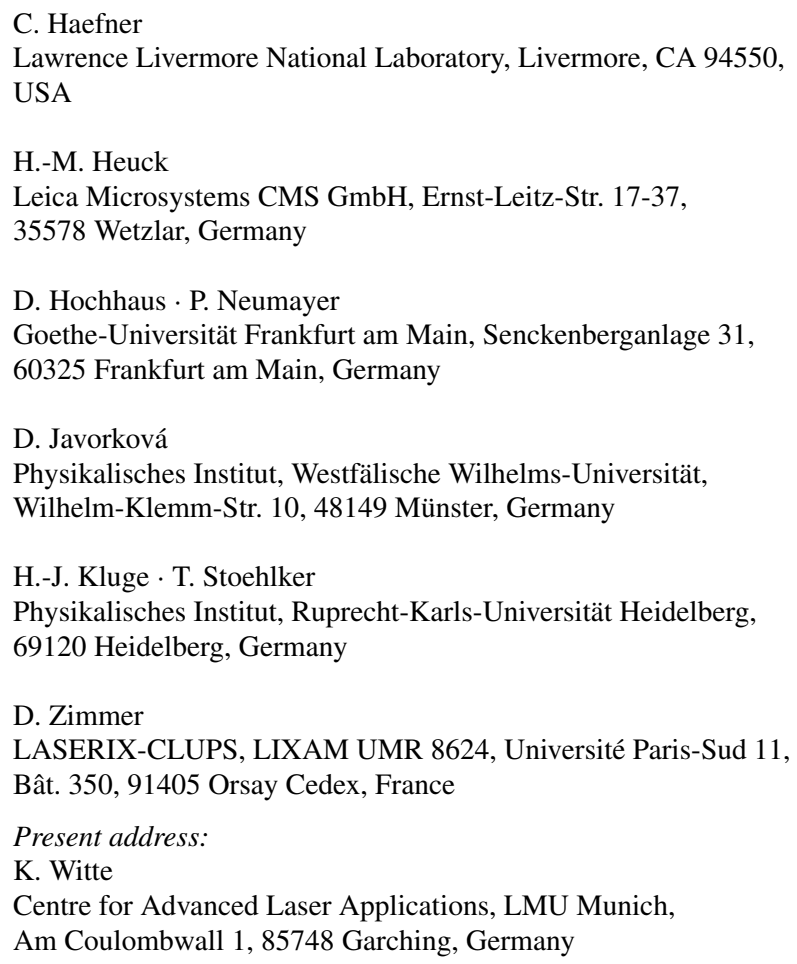


radiographic and spectral diagnostic setups, developments in the field of HEPW also show possible applications to emerging new fields like proton radiography, for instance. In these cases, the laser is used as a diagnostic tool that probes a sample prepared in a state relevant to HEDP or warm dense matter (WDM) studies. These states of matter are relevant to astrophysics as they represent conditions prevalent during supernovae explosion, in astrophysical jets or giant planet interiors.

At the Helmholtz-center GSI, heavy-ion beams are exploited to reach homogeneous WDM conditions for target of substantial sizes or as a probe to answer still-outstanding questions about energy loss of ions in plasma targets. These capabilities will be further enhanced when the new FAIR accelerator facility for antiproton research [5] will be commissioned. The interest in this field is in particular reflected in the large number of countries participating in the HEDgeHOB [6] and WDM [7] collaborations in charge of developing these experiments. A HEPW laser adds an essential diagnostic capability to the setups envisioned and justified the development of a Petawatt High Energy Laser for Heavy Ion Experiments (PHELIX) [8] in close proximity with the activities of GSI in the realm of plasma physics [9] and atomic physics [10]. In addition, recent developments in laser-generated particle acceleration open a new field of investigation at the interface between plasma physics and traditional accelerator physics.

In this paper, we report on the commissioning of the PHELIX facility and also on the first experimental results obtained at the facility. PHELIX is a hybrid Ti:sapphire/ $\mathrm{Nd}$ :glass laser system using large aperture amplifiers from the former Nova and Phebus laser systems at the Lawrence Livermore National Laboratory in the USA and the CEA Limeil-Valenton center in France, respectively. The laser serves three experimental areas that exploit the various capabilities of the machine. The facility is meant to be a useroriented facility where beam-time is granted based on the recommendation of an international advisory committee that meets and inspects experiment proposals on a regular basis. Additionally, PHELIX is a member of the integrated initiative of European laser research infrastructures, Laserlab [11], and offers beam-time as such to the scientist of European laboratories.

After a short overview of the laser architecture, the paper focuses on technological aspects that are unique to the PHELIX facility. Then, in the second part, a review of the first experimental results involving PHELIX is presented. This includes results obtained in combined laser and ion beam experiments on the ion stopping power of lasergenerated plasma, laser-generated coherent and incoherent $\mathrm{X}$-ray sources, and particle acceleration.

\section{Overview of the PHELIX facility}

The overview of PHELIX presented in Fig. 1 shows the schematic arrangement of the laser building and its neighboring experimental area (Z6) in the UNIversal Linear Accelerator (UNILAC) hall of GSI. The femtosecond front-end is used to generate the short sub-picosecond pulses. It consists of a commercial femtosecond laser oscillator (Coherent Mira) delivering pulses with a duration of $100 \mathrm{fs}$ and energies around $4 \mathrm{~nJ}$ at a repetition rate of $76 \mathrm{MHz}$. The oscillator is synchronized to an external clock using the low-jitter Synchrolock ${ }^{\mathrm{TM}}$ option from the same vendor. This external $76 \mathrm{MHz} \mathrm{RF}$ oscillator is also used to generate the timing signals of the facility, while a signal synthesizer is foreseen for future use with the GSI $108 \mathrm{MHz}$ reference clock. The pulse is temporally stretched to $2.3 \mathrm{~ns}$ full-width half maximum (FWHM) in a pulse stretcher whose architecture is a modified version of the stretcher architecture found in [1]. The pulse stretcher clips the spectrum at $12 \mathrm{~nm}$ because of the finite size of the optical grating used in its setup. The pulses are then amplified by two titanium-doped sapphire regenerative amplifiers with a repetition rate of $10 \mathrm{~Hz}$. The first one is set up in a linear configuration while the second one is a ring amplifier. The typical output energy after the ring regenerative amplifier is $30 \mathrm{~mJ}$. Using ultra fast Pockels cells the achievable intensity contrast ratio is better than $60 \mathrm{~dB}$.

For experiments requiring pulses in the nanosecond regime, a nanosecond front-end allows generating laser pulses with durations between 700 ps and 20 ns with programmable pulse shapes. The major part of the system is fiber-based, employing a commercial continuouswave $(\mathrm{CW})$ single-mode laser source (Koheras Basik), an acousto-optical modulator generating pulse slices of $100 \mathrm{~ns}$, a double-pass fiber amplifier and an intensity modulator which is driven by a programmable waveform generator (Tektronix AWG510). The fiber system delivers pulse energies up to $10 \mathrm{~nJ}$. A flash-lamp-pumped regenerative $\mathrm{Nd}$ :glass ring amplifier amplifies the pulses to energies up to $20 \mathrm{~mJ}$ with a repetition rate of $0.5 \mathrm{~Hz}$. The timings signals for the nanosecond front-end are generated locally at PHELIX during maintenance and alignment, but for experiments it is derived from the linear accelerator of GSI, leading to a synchronization of the ion and laser pulses typically better than 1 nanosecond.

Depending on the application the pulses generated by femtosecond front-end or the nanosecond front-end are then further amplified in the pre-amplifier. The pre-amplifier employs three flash-lamp-pumped Nd:glass amplifiers $(2 \times$ $19 \mathrm{~mm}$ diameter, and $1 \times 45 \mathrm{~mm})$. The input beam delivered by the front-end is stepwise magnified by Keppler telescopes up to a maximum exit beam diameter of $70 \mathrm{~mm}$ in order to keep the fluence within safe limits, below the damage threshold. Furthermore, a deformable mirror (Night $\mathrm{N}$ 


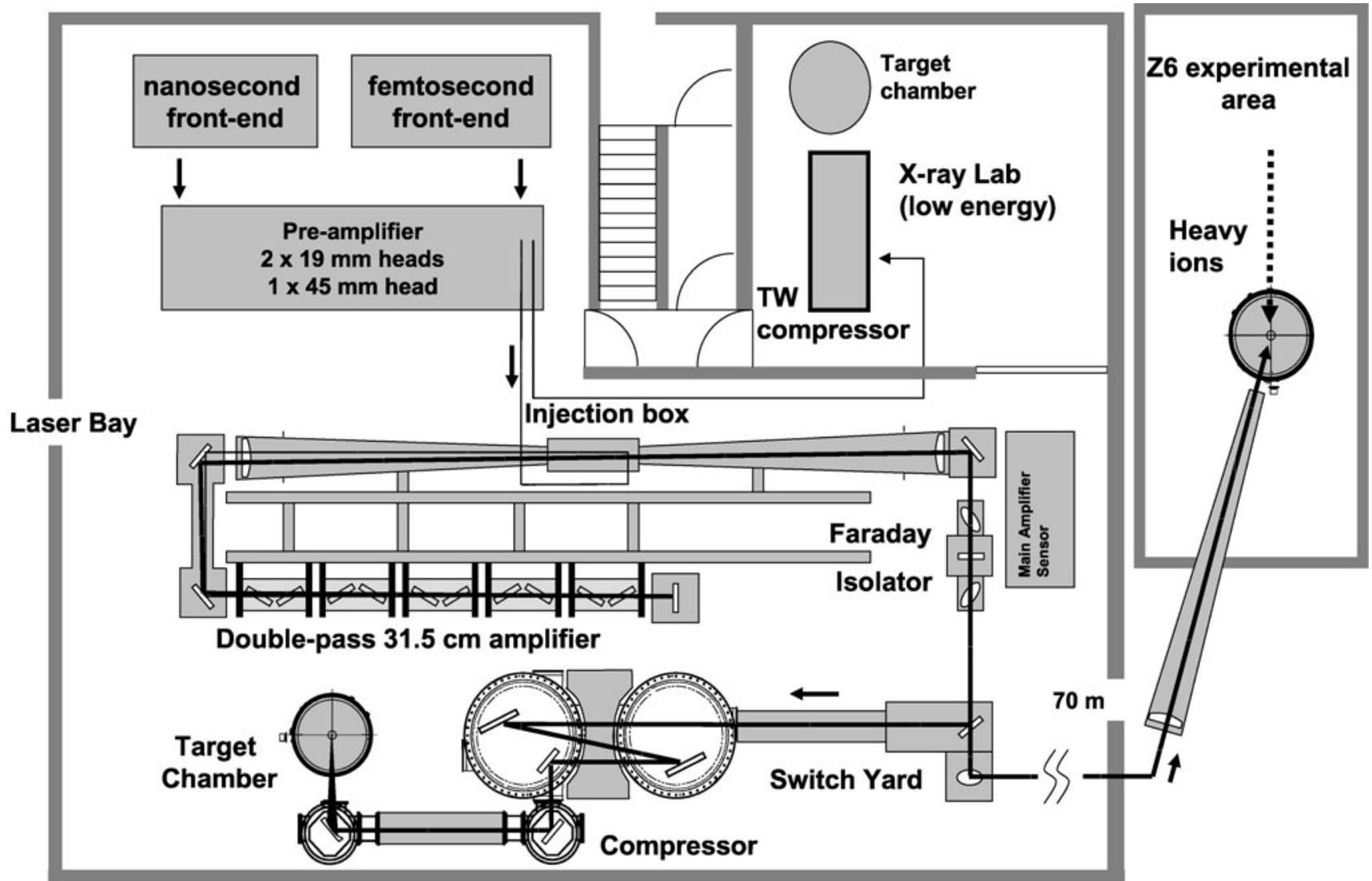

Fig. 1 Schematics of the PHELIX laser system. The beam is presently used at three different target stations: 1. X-ray lab; 2. Target chamber after passing the compressor; 3. Target station "Z-6" at the heavy-ion accelerator beam-line in the UNILAC hall

Ltd.), located at the end of the amplifier, can correct wavefront aberrations. This mirror is actively driven in a closedloop system, which utilizes a Shack-Hartmann sensor as a wavefront measurement device. Using this scheme, we successfully demonstrated correction of the on-shot aberrations of PHELIX, up to the end of the main amplifier, such that the spot profile available during alignment of the system corresponds accurately to the spot shape during the shots. After the pre-amplifier, the laser pulses of up to $10 \mathrm{~J}$ can be sent either to the X-ray lab, or to the main amplifier.

The main amplifier is set up in a double-pass configuration using five flash-lamp-pumped Nd:glass NOVA and PHEBUS amplifiers. The amplifier hardware was refurbished to make it compatible with the use in a CPA system. In particular, the $\mathrm{Nd}$ :glass slabs were re-polished to $\lambda / 10$ transmitted wavefront quality. The whole amplifier section was folded in order to fit within the building footprint. The double pass architecture is using geometrical separation of the incoming and outgoing pulses in the spatial filter. With amplifier modules having an individual clear aperture of $31.5 \mathrm{~cm}, 28-\mathrm{cm}$-diameter beams are successfully amplified and transported throughout the system; and the corresponding output energy available for the experiments exceeds $1 \mathrm{~kJ}$ for $10 \mathrm{~ns}$ pulses. This energy is only limited by the laser damage threshold of the Faraday isolator protecting the laser against possible back reflections.

At this point, the beam enters a switch yard where it can travel through a periscope and a telescope over 70 meters it to the UNILAC hall where combined laser and ion experiments can be done. Special care has been taken in designing the periscope mirror holders such as to avoid sagging, a source of astigmatism, and vibration, a source of pointing errors. At the Z6 target chamber, impact of the beam transport on the beam quality is minimal, while the pointing stability is about $10 \mu \mathrm{rad}$, peak-to-valley. The beam is focused into the target chamber using lens with a 4-m long focal length and a phase plate. In this way, a uniform intensity distribution is created over a $1-\mathrm{mm}$ large area, at a location where the ion beam has a $0.5-\mathrm{mm}$ diameter. The ion pulse and the laser meet with a jitter of $1 \mathrm{~ns}$, peak-to-valley. In addition, a second nanosecond laser beam generated by the nhelix laser [12] is available at this location, either as a three-color probe or a second heating source up to $100 \mathrm{~J}$. All three beams can be synchronized on the target with a jitter of $1 \mathrm{~ns}$ using the UNILAC $108 \mathrm{MHz}$ clock as a timing reference. 
Table 1 Present operational parameters of the PHELIX laser system. The temporal intensity contrast is given at $1 \mathrm{~ns}$ and $40 \mathrm{ps}$ before the main pulse reaches its maximum for the long pulse and short pulse, respectively

\begin{tabular}{lll}
\hline & Long pulse & Short pulse \\
\hline Pulse duration & $0.7-20 \mathrm{~ns}$ & $0.4-20 \mathrm{ps}$ \\
Energy & $0.3-1 \mathrm{~kJ}$ & $120 \mathrm{~J}$ \\
Max intensity & $10^{16} \mathrm{~W} / \mathrm{cm}^{2}$ & $10^{20} \mathrm{~W} / \mathrm{cm}^{2}$ \\
Repetition rate at & 1 shot every $90 \mathrm{~min}$ & 1 shot every $90 \mathrm{~min}$ \\
maximum power & & \\
Intensity contrast & $50 \mathrm{~dB}$ & $60 \mathrm{~dB}$ \\
\hline
\end{tabular}

As an alternative to the use in nanosecond regime at Z6, the short pulse capability of PHELIX can be exploited in the laser bay after the pulses are compressed. The pulse compressor uses multi-layer dielectric (MLD) gratings (Horiba Jobin-Yvon, France) which span $47 \mathrm{~cm}$ in the horizontal direction (dispersion) and $33 \mathrm{~cm}$ in the vertical direction. An incidence angle at the gratings of $72^{\circ}$ results in lower alignment and pointing sensitivity at the expense of a largely increased size of the compressor tank. In this geometry, the incident beam profile is horizontally limited to $12 \mathrm{~cm}$ due to the size of the gratings. In the vertical direction, the currently used beam extension is $24 \mathrm{~cm}$ so that the beam is elliptical. In order to achieve an optimal use of this area, the beam is shaped using lithographically produced serrated apertures combined with spatial filtering. This serrated aperture is located between the front-end and the pre-amplifier. The maximum output energy after the pulse compressor is limited by the damage threshold of the final grating. For 10- to 50-pslong pulses, the maximal throughput energy is $200 \mathrm{~J}$. For the shortest pulses, below 1 ps duration, it is reduced to $120 \mathrm{~J}$. This corresponds to a nominal peak power of $300 \mathrm{TW}$ for $400 \mathrm{fs}$ pulses. An overview of the laser parameters can be found in Table 1 that summarizes the laser output characteristics as a function of the type of operation (long or short pulse).

The operation of the whole laser facility, which consists in the routine alignment checks and the control of the shot sequence from the control room, is centralized by the PHELIX control system (PCS). The PCS is based on an inhouse-developed control system framework also in use in other places [13], and it uses an object-oriented approach implemented with the LabVIEW programming language. Currently running distributed on 20 computers, this eventdriven software architecture is, in addition, readily scalable. About 10,000 process variables are handled to guarantee the safe operation of the laser. Practically, this corresponds to the remote operation of more than 50 actuators and 25 cameras for the alignment of the laser beam, loading and firing the capacitor banks of the pre-amplifier and main-amplifier flash-lamps, the collection of the laser parameters during the shot (e.g., pulse energy, pulse profile, spatial energy density distribution in the near and far fields), and the automatic handling of interlocks and timing signals relevant to a machinesafe operation of the facility.

\section{Commissioning of the main amplifier section}

The main amplifier section of the laser is based on flashlamp-pumped Nd:glass amplifier modules. These and various other assemblies such as telescope lenses or mechanical mounts were formerly in use in the Nova and Phebus laser facilities. They had been designed for use with long pulses and in loose focusing geometries. However, the requirements associated with the design of a modern HEPW laser system put more constraints on these elements. In particular, the maximum allowed transmitted wavefront aberrations of the amplifier discs required an extensive refurbishment which included a re-polishing of the amplifier slabs to meet the specifications. Furthermore, the 530-mm-diameter telescope lenses received an improved anti-reflective coating pushing down the residual reflectivity to less than $0.6 \%$. This was necessary because of the operation of the amplifier chain in double-pass configuration, were possible back reflections could easily become harmful to the system. A Faraday isolator located after the amplifier completes the laser system by shielding it against back-reflected light, and a rotating large aperture half-wave plate allows one to adjust the polarization direction of the light.

During 2006, most electrical tests in combination with checks of the control and safety systems were performed. At the end of 2006, a first experiment was carried out using the main amplifier section in a single-pass configuration, which resulted in (uncompressed) pulse energies of up to $40 \mathrm{~J}$. At the beginning of 2007, the final double-pass configuration of the amplifier was commissioned, terminating the beam on a calorimeter. The maximum pulse energy in excess of 500 Joules obtained with 600 ps long pulses demonstrated the target value for the amplifier gain (100) at a capacitor bank voltage of $18 \mathrm{kV}$. Following the installation of the large-aperture Faraday isolator and the subsequent transport beam line to the UNILAC experimental hall, the first shots on target were successfully performed in May 2008. In this experiment, the precise and reliable synchronization of PHELIX with the ion beam was demonstrated. The following efforts were focused on the commissioning of the pulse compressor and the installation of the target chamber for laser-only experiments in the PHELIX building. First compressed pulses were delivered to an experiment in October 2008, with a pulse length of $700 \mathrm{fs}$ and energies up to $100 \mathrm{~J}$. A plot of the energy of the full system shots as a function of the main amplifier input energy is presented in Fig. 2. The data correspond to the case when the system 


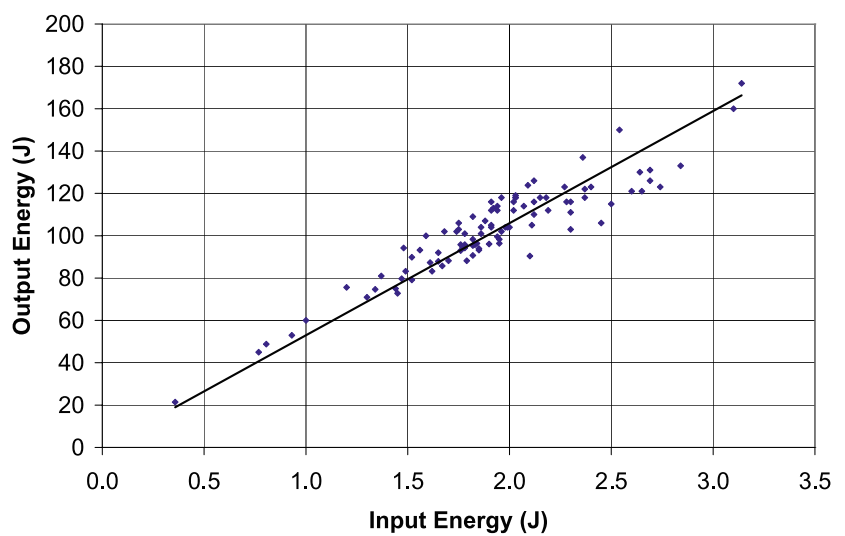

Fig. 2 Output versus input energy of the main amplifier section

is operated with short pulses ( $<1 \mathrm{ps})$ delivered by the femtosecond front-end, and when the main amplifier was shot at a capacitor bank voltage of $15.5 \mathrm{kV}$. A linear fit delivers a gain of $53( \pm 4.6)$ for broadband pulses with a variable spectral width centered at $1054 \mathrm{~nm}$. The spread in the output energy is mainly determined by slight deviations in the pulse spectrum between data sets from different measurement campaigns. We attribute the deviation to the average to the wavelength-dependent gain of the Nd:glass material. The constant gain behavior furthermore shows that the system is still operated in the linear amplification regime with no observable saturation effects. In case of operations with the long pulse (10 ns) created by the nanosecond front-end, the average gain is higher, at a value equal to 63 . In such a case, the 500-MHz-wide laser-line width is much smaller than the 20-nm-wide stimulated-emission cross-section of the amplification medium. The wavelength of the seeding laser was therefore adjusted, by design, to match the peak of the spectral gain of the amplification material.

In addition to the refurbishments of the original components, it was necessary to improve the capabilities of the main amplifier beam-analysis sensor so that it can be used for precise laser beam diagnostics. Besides the beam energy and the near-field intensity distribution, operation of the system requires the recording of the laser spectrum, the temporal shape, as well as the measurement of the wavefront (Shack-Hartmann sensor) and the far-field distribution with high resolution. For reliable wavefront measurements the aberrations of the internal beam path of the sensor itself must be well-known and understood. For this purpose, the sensor path was carefully characterized in double-pass configuration using a reference point source. The use of compensated multi-lens telescopes, de-magnifying the incoming 30-cmwide beam down to some millimeters, drastically reduced the overall static aberrations. In addition, great importance was attached to the quality of insertable optical attenuators which must cover a dynamic range of $10^{6}$.
Precise control of the shot energy plays an important role for the experiments. Furthermore, a well-defined, homogeneous distribution of the spatial beam fluence is required to avoid local intensity maxima which might destroy optical components. The targeted spatial intensity distribution is a high-order super-Gaussian profile, which is generated using a lithographically manufactured serrated aperture located at the input of the pre-amplifier in combination with a properly matched spatial frequency filter. The quality of the beam can be expressed in terms of the fill factor (FF). The fill factor is the intensity distribution integrated over the convex hull area of the beam above a threshold level divided by the same integral of a top-hat beam:

$$
F F=\frac{\int_{A} I(\vec{r})}{A I_{\operatorname{Max}}},
$$

where $A$ is the convex hull area of the beam with intensities above a threshold level, $I(r)$ is the intensity at the position $r$, and $I_{\mathrm{Max}}$ is the maximum intensity within $A$. A fill factor of 1.0 therefore corresponds to a perfect top-hat beam while all non-uniformities lead to values below one. In particular, this value significantly drops in the presence of small high intensity areas (hot spots) which might locally exceed the laser damage threshold of optical components. For an arbitrarily-chosen intensity threshold level of $13 \%$ of $I_{\text {Max }}\left(1 / e^{2}\right)$, PHELIX currently achieves regularly fill factors up to 0.69 (Fig. 3), which come close to the theoretical limit for super-Gaussian profiles. The decision to use of the $13 \%$ threshold is based on the necessary trade-off between experimental limitations imposed by the use of 8-bit cameras for the measurement and the wish to take into account the largest possible surface area.

The most critical optical component of PHELIX in terms of laser damage threshold is the Faraday isolator of the main amplifier section. The active material responsible for the rotation of the polarization plane is terbium-doped glass. Its damage threshold fluence is $2 \mathrm{~J} / \mathrm{cm}^{2}$ for $1 \mathrm{~ns}$ pulses. With a safety margin of $50 \%$, this limits the maximum energy for a stretched femtosecond pulse with a diameter of $28 \mathrm{~cm}$ to $615 \mathrm{~J}$. However, this is only valid for a fill factor of 1 . The maximum energy for a typical PHELIX beam is reduced by this factor $(<0.65)$ leading to a maximum output energy of $400 \mathrm{~J}$. For long pulses, with durations around $10 \mathrm{~ns}$, the (scaled) damage threshold is considerably higher $\left(6 \mathrm{~J} / \mathrm{cm}^{2}\right)$. Hence PHELIX is able to safely deliver output energies in excess to $1 \mathrm{~kJ}$ with $10 \mathrm{~ns}$ long pulses.

\section{Short pulse control}

Many experiments planned with PHELIX rely on the ability to create and amplify short laser pulses in the sub-picosecond regime. The Chirped-Pulse Amplification 
Fig. 3 Near-field intensity distribution in the case of a round (a) and elliptical (b) beam used in the long- and short-pulse experiments, respectively. The white contour line represents the convex hull of the area used in the fill factor calculation. The fill factor compares to a maximum theoretical value of $89 \%$ for the targeted beam shapes
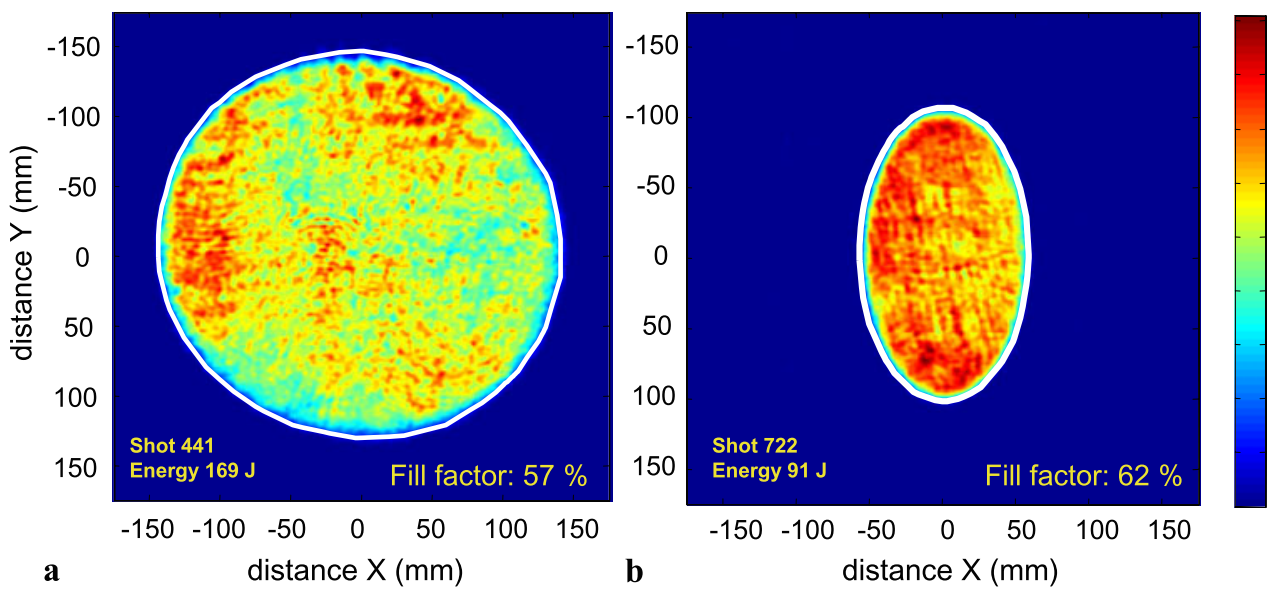

(CPA) [14] is the standard technique used to perform this and it is employed in the PHELIX system. In this scheme, the short laser pulses are temporally stretched before the amplification, such as to avoid accumulation of nonlinear effects during amplification. After final amplification and just prior entering the interaction chamber they are recompressed to their original duration. Particular to PHELIX are: first, the large stretching factor necessary to reach a high energy; second, the gain narrowing management necessary in a system that uses $\mathrm{Nd}$ :glass as a main amplification medium; and third, spatio-temporal effects originating from the amplification of large-diameter laser beams.

Large stretching factors can be reached using various stretcher designs. Based on a design previously reported elsewhere [1], the stretcher at PHELIX utilizes a 30-cm diameter primary mirror and one 1740 lines/mm grating used at 72 degree incidence angle. The stretcher distance of $4 \mathrm{~m}$ yields a stretching factor of $190 \mathrm{ps} / \mathrm{nm}$ and stretched pulses of $2.3 \mathrm{~ns}$ duration. One requirement common to all stretchers is the excellent surface quality of the optics used in the setup [15]. The stretcher at PHELIX utilizes spherical and flat mirrors with an optical surface quality better than $\lambda / 100$ in a root-mean-square (rms) sense. This is done so that the spectral phase error introduced by the stretcher stays well below $\pi$ and does not perturb the final pulse compression. The optical assembly of the stretcher is located on a translation stage which allows an operator to easily adjust the pulse duration up to 20 ps using the motorized stage and pre-set positions. This offers many advantages over the standard technique (adjusting the compressor grating-to-grating distance), which quickly becomes unpractical with grating assemblies that weigh $100 \mathrm{~kg}$. To characterize the stretcher and the femtosecond front-end in general, an auxiliary compressor using one 1480 lines/mm grating in a folded configuration has been used. Our calculations demonstrated that the residual spectral phase, originating from mismatched grating groove densities in the stretcher and compressor, is minimal at pulse durations exceeding $100 \mathrm{fs}$ as it is the case

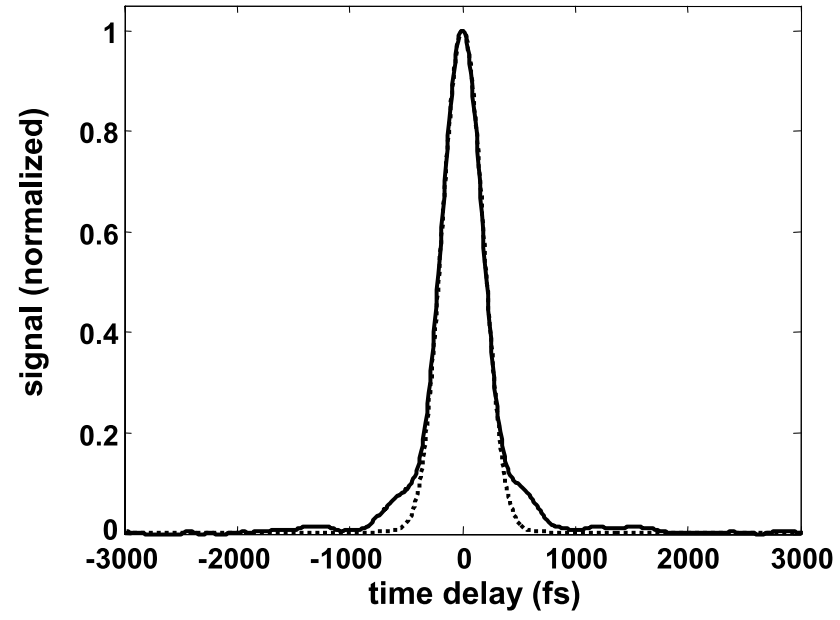

Fig. 4 Single-shot autocorrelation measurement of the short pulse after the auxiliary compressor (solid line) and theoretical autocorrelation (dashed line) calculated from the pulse spectrum

at PHELIX. As shown in Fig. 4, the pulse duration of the short pulses generated by the femtosecond front-end can be re-compressed at durations close to their Fourier-transform limit.

The second point which has to be taken into account is the reduction of the spectral bandwidth of the laser pulse (known as gain narrowing) during the amplification. While the first part of the amplification uses Ti:sapphire as a broadband amplification medium at $1054 \mathrm{~nm}$, the second part of the amplifier relies on Nd:glass, which seriously limits the width of the amplified pulse. A solution that mitigates this effect relies on the use of a birefringent filter [16] to shape the spectrum of the laser such as to compensate for the gain narrowing occurring in the system. The spectral filtering is done between the two regenerative amplifiers so that the energy losses induced by the filter are compensated in the second amplifier. After this stage, the spectrum shows a dip at the central gain wavelength of Nd:glass, at a wavelength of $1054 \mathrm{~nm}$. At the end of the system, this dip is re-filled and 


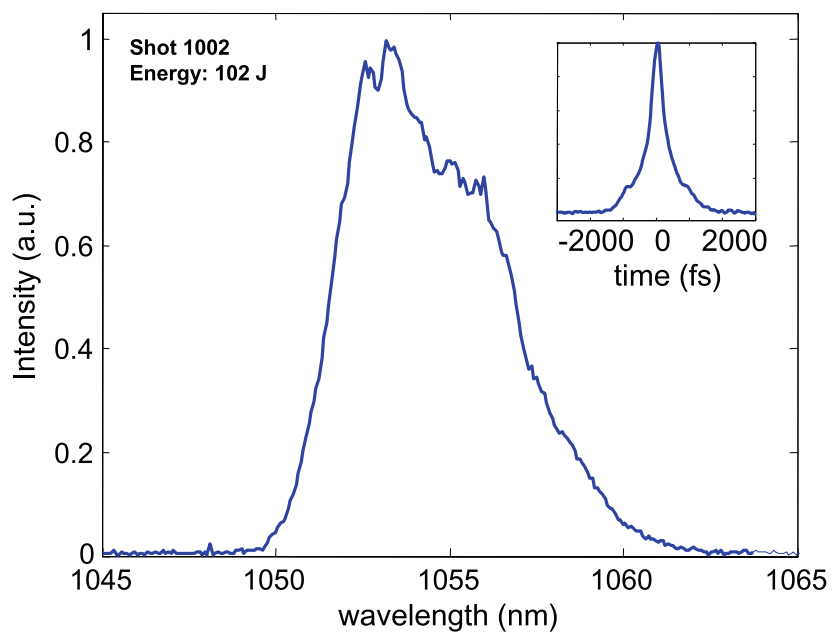

Fig. 5 Output spectrum of the laser pulse measured as it leaves the main amplifier. The spectral width is $5.3 \mathrm{~nm}$ FWHM and corresponds to a $370 \mathrm{fs}$ Fourier-transform limited pulse. In the insert is a single-shot autocorrelation trace of the pulse at this particular shot. Besides large wings, the 490 -fs autocorrelation FWHM is within 5\% of the Fourier-transform limit

the effective spectral width is increased. Figure 5 shows the output spectrum of the laser when the birefringent filter is used. The width of the pulse spectrum does not exceed $3 \mathrm{~nm}$ FWHM when no pre-compensation is applied. But when the birefringent filter is used, pulses with stable spectra in excess of $5 \mathrm{~nm}$ FWHM are typical for PHELIX on a daily basis. The effect on the Fourier transform limit of the laser pulse is important, reducing the pulse duration from values above $600 \mathrm{fs}$ to below $400 \mathrm{fs}$.

The spatio-temporal effects can have a relatively large impact on the pulse compression in a large-beam CPA system [17], and these effects require careful analysis of the pulse profile over the whole aperture of the beam. A complication to that is the need to perform the measurement during alignment with a few millijoules as well as on-the-shot with several hundreds of joules. For this, we have developed a down-collimating system that not only adapts the beam size to the entrance pupil of most measurement device but also manages the energy delivered to the diagnostic package without an excess of B-integral accumulation. A view of the setup can be seen in Fig. 6. The diagnostic beam used for this is the leakage through the diagnostic mirror, which was manufactured so that its transmitted wavefront is not only of high quality but the transmitted wavefront of the first internal reflection is also usable. This condition is fulfilled when the glass substrate is void of any defect and the surface polished to a perfect flat. The interest lies in the fact that the beam created by internal reflection in the substrate caries an energy $4.5 \times 10^{-4}$ lower than the main pulse energy and can therefore propagate without a significant B-integral accumulation through material. The pulse intensity is then further controlled using five partially transmitting plates that can be remotely inserted into the beam. During the alignment phase, only the direct transmission through the leaky mirror is used. Both low-energy and high-energy channels are recombined after the beam is down-collimated as shown in Fig. 6. Finally the diagnostic package uses a home-built high-dynamic-range single-shot cross correlator [18], that is used to cover the wide range of possible input energies. Here the large dynamic range is used not so much to gather information with a high dynamic range but rather to ease operation of the device that can then still tolerate a large variation of the input energy.

In addition to developing diagnostics and optimizing the short-pulse profile, we also developed a series of pulsecontrol tools required for some experiment. The femtosecond front-end is hosting, for instance, a Mach-Zehnder interferometer that allows the creation of a pre-pulse of known duration and energy. The pre-pulse duration can be set independently from the main short pulse using a small compressor located in one of the arms of the interferometer [19]. Additionally, we use the fact that the compressor is using two gratings in single pass to introduce a pulse front tilt to the beam when necessary.

The last point concerning the temporal aspects of PHELIX deals with the temporal contrast of the laser pulses. Third-order cross-correlation measurements of the laser pulse show a typical pulse pedestal at the level of $10^{-6}$, most likely due to amplified spontaneous emission (ASE). This pedestal extends for up to 1 to 2 nanoseconds before the pulse. Particular to our setup was a large number of pre-pulses originating from the cross-talk between the three laser-cavities used in the front-end. The solution around this problem has been to isolate the short pulse oscillator and the subsequent two amplifiers from each other using Pockels cells. In particular, a fast Pockels cell driver is used at the output of the femtosecond front-end to remove pre-pulses located 1 and 3 nanoseconds before the main pulse. As a consequence, we cannot detect any pre-pulse with an energy higher than $100 \mu \mathrm{J}$, which is the detection limit of our measurement scheme.

\section{Cost-effective beam focusing}

The final focusing optics assembly in a HEPW laser system is facing contradictory issues. On the one hand, the use of short pulses, which prevent from using transmission optics, requires that one employs a demanding off-axis optical system. The solution based on the traditional polishing of an optical substrate yields long-procurement times and expensive pieces that are often unique. On the other hand, the conditions close to the target, at the time of the shot, are so harsh that the focusing optics is often damaged by debris and secondary radiation, requiring its regular replacement or extra care for its use. 


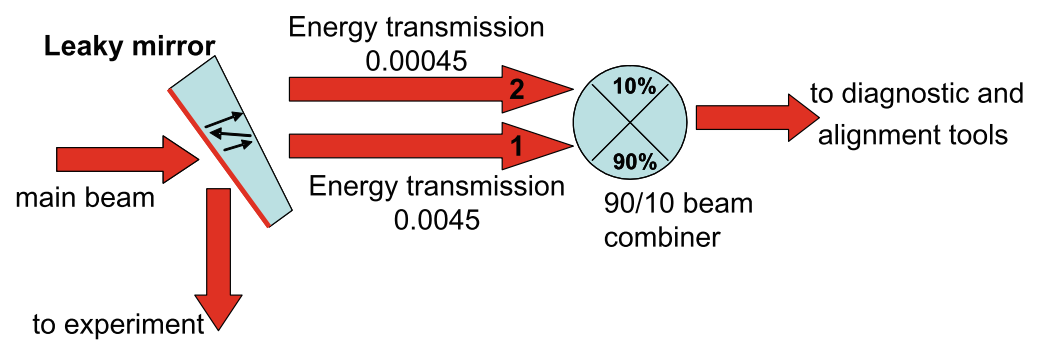

Fig. 6 Schematics of the short-pulse diagnostic beamline showing the energy attenuation scheme. During the alignment phase, the beam directly transmitted through the leaky mirror is used with an attenuated energy of $4 \times 10^{-3}$. At the time of the shot, the beam generated by

Thanks to recent progress in diamond turning, we were able to demonstrate that a diamond turned off-axis parabola made of copper can be successfully employed for an extended period of time in standard experiments. The advantages of a metallic mirror are manifold. First of all, the costs and lead time for such optics is drastically reduced by roughly one order of magnitude. Second, the constraints in geometrical arrangement possibilities offered by diamond turning are not these of traditional polishing. For instance, a $90^{\circ}$ large-aperture parabola is not more complicated to manufacture than a standard spherical shape, while the complications met by traditional polishing techniques would normally forbid such an optical arrangement. Other quadric surfaces of any numerical aperture can also be conceived for an aberration-free imaging without much increase in the manufacturing complexity. And third, the use of metallic substrates offers the possibility to collect the back-reflected light over a large spectrum, allowing for additional target diagnostics opportunities in the visible and near infrared spectral bandwidths. However, the overall performance of a diamond-turned piece of optics does not reach that of traditionally polished ones and some trade-off have to be made in order to reach a performance compatible with the use in a high-energy laser system. In particular, the surface error and its roughness have to be mitigated.

We worked together with a company (Kaleido, Denmark) to develop a parabola suitable for our application. A feature of the surface is the periodic structure left by the tool during the machining process. This can be seen in the insert at the top left of Fig. 7 that shows a measurement of a sample on a microscopic scale. The period and amplitude of the surface structure is determined by the machining speed and a surface roughness as low as $5 \mathrm{~nm}$ rms can be obtained at lower machining speeds. The effect of the roughness is a spread of the energy out of the main central spot of the laser. The amount of energy present in the central spot can be easily estimated in the frame of the Fresnel diffraction, where the far-field energy distribution in the central spot is related to the Fourier transform of the disturbed wavefront, internal reflection in the leaky mirror is used with an attenuated energy of $5 \times 10^{-5}$. The beam combiner allows for easy use of one or the other of the two beams while providing an additional attenuation factor. This setup keeps the B integral below 1 for pulses up to $500 \mathrm{~J}$ in $500 \mathrm{fs}$

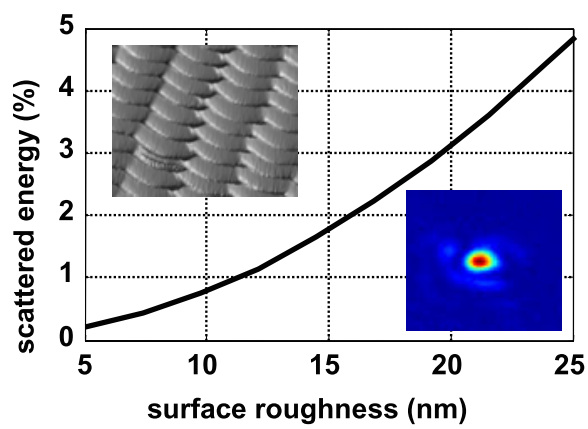

Fig. 7 Calculated scattered energy as a function of the surface roughness for surfaces modeled using scaled periodic tooling imprints (upper left insert) on the surface. The lower right insert is a measurement of the energy distribution at the focus of the parabolic mirror, showing a $16 \times 12 \mu \mathrm{m}^{2}$ focal spot

taken at the origin. Figure 7 shows a plot of the estimated energy losses due to surface scattering as a function of the surface roughness, using the hypothesis that the tooling marks scale with the surface roughness. As shown, energy losses well below $5 \%$ can be obtained for values of the surface roughness well above the technical limits of the turning machine.

In addition to tolerable scattering losses, the beams focused by the copper parabola offer qualitatively good results: the laser far-field distribution at the target chamber and that measured after the compressor using the compressor sensor package are showing similar shapes when the parabola is aligned to best focus. This indicates that the wavefront error introduced by the parabola is practically negligible. Strehl ratio measurements of the far field distribution of energy of the beam regularly show values at about 0.3 , which is a good value considering that no adaptive wavefront correction is necessary to achieve this goal. These measurements, together with experimental evidence of high on-target intensities (see below), allow us to infer that diamond-turned final focusing systems offer an interesting alternative to standard optics. 
Fig. 8 Experimental set up for the investigation of the interaction of ions with laser driven plasma

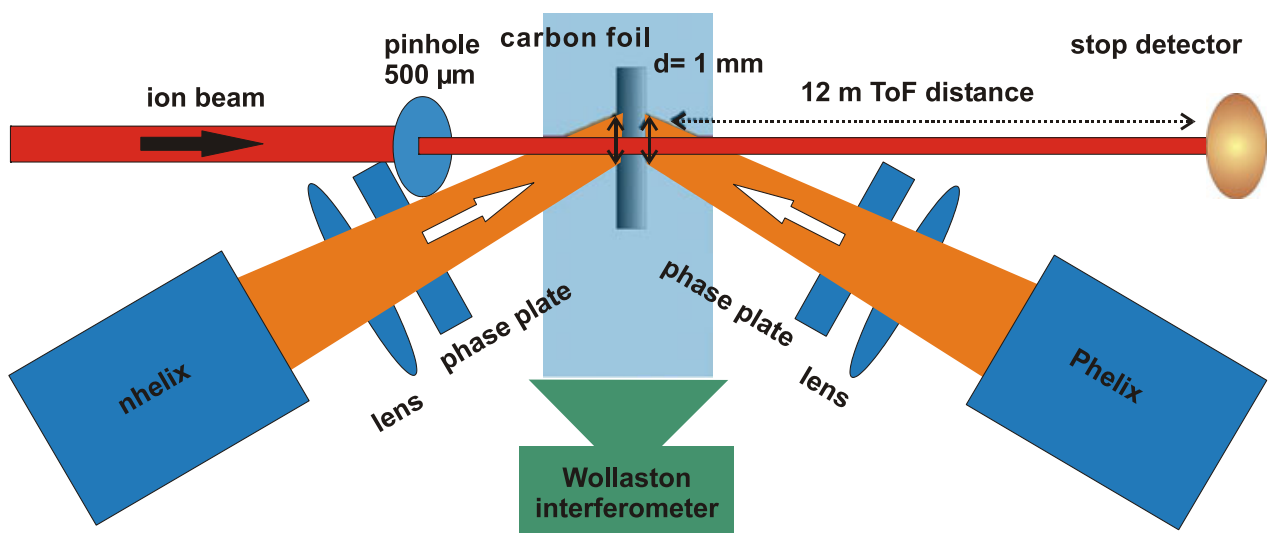

undisturbed beam

disturbed beam

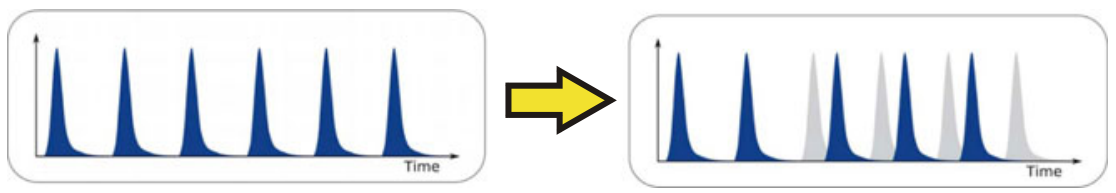

\section{Experiments with ions and nanosecond pulses}

A central line of research of the plasma physics group at GSI concerns the interaction of energetic ion beams with plasma matter. The unique combination of powerful laser systems and an intense ion beam opens the possibility to investigate the energy deposition and charge state distributions of swift heavy ions penetrating laser generated plasma, a subject of interest for the astrophysics, inertial fusion or fundamental research.

Earlier experiments, where ions were penetrating gas discharge or Z-pinch plasmas [20, 21] have shown a significant increase of both stopping power and mean charge state of the projectile compared to the interaction with cold matter. However, only few experimental data exist and they are limited to low free electron densities $\left(n_{e}<5 \times 10^{19} \mathrm{~cm}^{-3}\right)$ and low plasma temperatures $\left(T_{p}<20 \mathrm{eV}\right)$. The GSI plasma physics group has dedicated its efforts to extend the experimental data base for the stopping power of ions in plasmas to higher densities and temperatures.

An important step to enable such experiments was the completion of the laser beam transport to the heavy-ion target station Z6, a branch of the linear accelerator UNILAC at GSI. In 2008, first successful experiments exploited lasergenerated plasma targets for interaction experiments with heavy ions. A new experimental setup, using PHELIX additionally to the already existing nhelix laser [12], has been set up (see Fig. 8) and it quickly showed a significant improvement in the quality of the measured data.

There, a thin foil, mainly a carbon foil with a thickness of $0.5 \mu \mathrm{m}$, is irradiated by the PHELIX laser as well as the nhelix laser from both sides, transforming the foil into a dense and hot plasma reaching densities of $1 \%$ of solid density and temperatures of 100-200 eV. To keep the plasma as homogeneous as possible for the ion beam, restricted to a $500-\mu \mathrm{m}$ diameter beam by a pinhole, a random-phase plate located in the laser beam produces a 1-mm-large focal spot with a "top hat" spatial intensity distribution. Under these conditions, the plasma expansion in the central region is quasi one-dimensional during the first few nanoseconds. In earlier experiments, where only the nhelix laser was irradiating the foil from one side, the heating of the foil to a plasma state was taking too long compared to the characteristic time of the plasma expansion. Furthermore, the plasma was inhomogeneous because of the microscopic inhomogeneities in the laser intensity distribution of the focal spot generated by a random-phase plate; and the ablation pressure pressed the foil back for several hundreds of $\mu \mathrm{m}$. The new set up using PHELIX irradiating the foil from the opposite side offers several advantages: first, the foil is transformed to the plasma state faster; second, the very thin foil remains in the center so the ablation pressure differences from both sides are reduced; and third, the inhomogeneity caused by the two phase plates are smeared out, resulting in significantly more homogeneous plasma. As this plasma is expanding, density and temperature gradients develop on both sides; and these characteristics have to be experimentally measured, e.g., by laser interferometry. At the same time, an ion pulse with a length of a few hundred $\mu$ s, built up of micro bunches with a length of $3 \mathrm{~ns}$ FWHM and a frequency of $108 \mathrm{MHz}$, is probing the plasma in a direction perpendicular to the initial foil. This means the expanding plasma is being investigated each $9.2 \mathrm{~ns}$. The relative delay between the laser and the ion bunches can be shifted with an accuracy of $1 \mathrm{~ns}$. 
A time of flight measurement characterizes energy of the ions penetrating first of all the cold foil, followed by dense and hot plasma, which is expanding and cooling down until all matter has vanished and the ions fly through vacuum with their initial energy. Any change of the stopping power, as it occurs, e.g., when the laser heats up the target to the plasma state, can be detected as a deviation from the regular $108 \mathrm{MHz}$ periodic signal (see inlay in Fig. 8) [22]. A dipole magnet behind the target chamber can be employed to measure the charge state distribution of each single ion bunch after exiting the plasma. As a result, we obtain a set of energy loss data and charge state distributions of ions interacting with plasma. From these results, knowledge about the fundamental interaction mechanisms, like ion-energy deposition and ion-charge transfer reactions in plasma, can be obtained. To our knowledge, GSI is the only place worldwide where these kinds of experiments can be performed.

\section{Experiments with short pulses: protons and X-rays}

Using intense pulses of energetic heavy ions, plasma states relevant at the Earth center and inside giant planets like Jupiter can be prepared in the laboratory and probed by advanced laser diagnostics. A central point of the research interest at GSI is the properties of WDM, which is a highly coupled plasma state at close to solid density and temperature between 1 and $100 \mathrm{eV}$. Central questions like the location of critical states and phase transitions under such extreme conditions will be in reach of experiments. The role of PHELIX is in this case to supply novel diagnostic approaches using laser generated X-ray and particle beams.

Interaction experiments have been performed using the PHELIX facility as early as 2006. Since 2007, an experimental program involving national and international user groups is established. These groups exploit the possibilities offered by the experimental places in the X-ray laboratory, the laser bay and the Z6 experimental area.

One of the fields that receive attention in the PHELIX science program is that of laser-generated soft-X-ray lasers. On the way to realization of soft X-ray lasers close to the water window, a main obstacle is the high pump laser energy required for the preparation of the lasing medium and the complexity of the setup. In 2008, we demonstrated the concept of the double-pulse pumping non-normal incidence geometry that allows for a drastic simplification of the experimental setup [19, 23]. Recently, we developed this elaborate pumping scheme further, and we applied it to higher pumping energies in the framework of short-wavelength soft $\mathrm{X}$-ray lasers. For that, an optimized double-pulse structure is created in the front-end of the laser system, which then propagates collinearly through the amplifiers and the compressor and strikes the target under optimized non-normal incidence. In that way, X-ray lasing is obtained at a low energy threshold while the complexity of the setup is dramatically reduced. The optimized double pulse consists of a $200 \mathrm{ps}$ long pre-pulse that precedes the 1-ps long main pulse by $130 \mathrm{ps}$. The laser beam is focussed to a line with $10 \mathrm{~mm} \times 20 \mu \mathrm{m}$ FWHM, on a slab target under non-normal incidence of 17 degrees with a travelling-wave speed of 1.1 times the speed of light, creating the transient collisionally excited gain for the soft X-ray laser transition. The focusing system consists only of one 90-degree off-axis copper parabola with a diameter of $300 \mathrm{~mm}$ and a focal length of $1500 \mathrm{~mm}$, producing the line focus via the aberration by the 4 degree tilt off the normal off-axis incidence angle. The travelling wave is tuned by turning the second grating of the PHELIX two-grating compressor. One can show that a spatially dependent additional phase term is added to the pulse, meaning that the compression is not spatially uniform. However, when the beam is large compared to the spectral spread on the grating, the increase in pulse duration at the edge of the beam is minimal. For instance, a 0.17 degree grating tilt yields a $30 \mathrm{ps}$ delay at the focus when a $12 \mathrm{~cm}$ beam is used, but at the non-detrimental expense of an increase in the pulse duration by 250 fs on each side of the beam. During the alignment phase, a test plate with two holes separated by $10 \mathrm{~mm}$ is located at the target plane. The two beams are then focused into a nonlinear crystal were second harmonic light is generated. The two-pulse front-end is used at this point to control the amount of pulse-front tilt introduced by the compressor. Practically, this corresponds to turning the grating in the compressor until the delay, necessary for getting a signal, equals $30 \mathrm{ps}$ or $15 \mathrm{~mm}$ on the delay stage of the two-pulse setup. Details about the experimental setup and traveling-wave calculation are beyond the scope of this paper and will be presented in another publication.

We obtained lasing of Ni-like samarium X-ray laser at $7.36 \mathrm{~nm}$ with a total pump energy as low as 36 joules [24]. Figure 9 shows a measurement of the spectrum used as a signature of the X-ray emission. The X-ray laser line is clearly visible in the first and second order of the spectrometer. This result demonstrates the efficiency of the setup, as this pump energy is just half of the values required in previous experiments. This is an important milestone for applying the pumping scheme also for higher pumping pulse energies, which are necessary for reaching soft X-ray laser wavelengths close to the water window. In addition, the reduction of the total pump energy now makes X-ray lasers also available at this wavelength for high repetition-rate lasers like the LASERIX facility.

A key application of PHELIX in combined experiments is to enable X-ray diagnostics of high-energy density states of matter produced with intense heavy ion beams from GSI or FAIR. This requires multi-keV X-ray energies to penetrate the dense plasma [25]. The development of laser driven 
Fig. 9 Spectrum of a Ni-like samarium X-ray laser obtained with a flat-field spectrometer. The lasing line at $7.36 \mathrm{~nm}$ is visible in the first and second order of the spectrum

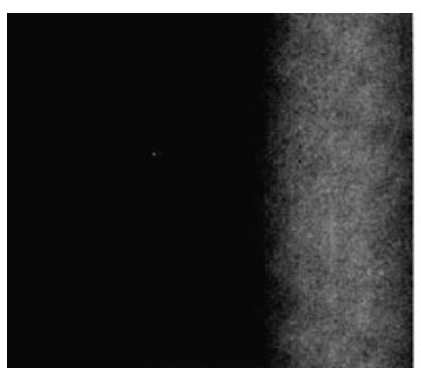

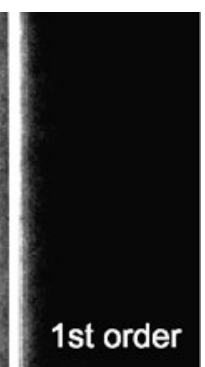

high-brightness fast X-ray sources is therefore a research activity that constitutes a key application of PHELIX. The development of backlighter sources at GSI focuses on highintensity short-pulse driven sources due to their favorable scaling to higher photon energies. When light is focused onto matter to relativistic intensities, a large fraction of the laser energy is converted into super-thermal electrons accelerated to $\mathrm{MeV}$ energies. These electrons propagate through the target material and emit high-energy bremsstrahlung when scattering off target atoms. In addition they ionize strongly bound inner-shell electrons of the target atoms giving rise to characteristic line emission. High-intensity shortpulse-driven X-ray sources possess excellent characteristics for diagnostic purposes. The duration of the emission is closely linked to the hot-electron relaxation and is on the order of few picoseconds. Spatially, the emission is confined to the solid density part of the target. Using thin foil or wire targets, source sizes as small as 10 microns are realized, enabling high-resolution point projection radiography [26] with picosecond time resolution. In recent experiments at PHELIX, we have optimized a $\mathrm{Cu} \mathrm{K}$-alpha backlighter source. Thin foils and wires with dimensions of few tens of microns were irradiated, and K-alpha and hard X-ray emission was determined quantitatively as function of the laser parameters pulse duration and focal spot size over a wide range of focal intensities.

A variety of X-ray diagnostics/spectrometers is employed in these experiments. A single photon counting camera gives a broad overview of the X-rays produced from about $5 \mathrm{keV}$ to over $20 \mathrm{keV}$. The setup employs a cooled back-illuminated CCD camera $(1024 \times 1024$ pixel $)$ viewing the target at a distance of about $3.3 \mathrm{~m}$ through a thin beryllium window. An evacuated pipe avoids absorption in air allowing detection of X-rays below $10 \mathrm{keV}$. Filtering and distance are chosen to operate in the single photon mode, so that on average less than 1 photon per pixel is detected. The device is absolutely calibrated using a radioactive source. Post-processing selects single pixel events. A single detection-event probability of about $3 \%$ is determined for the calibration energy $(5.9 \mathrm{keV})$.

Figure 10 shows a spectrum obtained from a $100 \mathrm{~J}$ laser shot. Up to $10^{13} \mathrm{Cu} \mathrm{K}$-alpha photons were generated per shot, corresponding to a conversion efficiency of laser energy into line radiation of the order of $10^{-4}$, which is typical
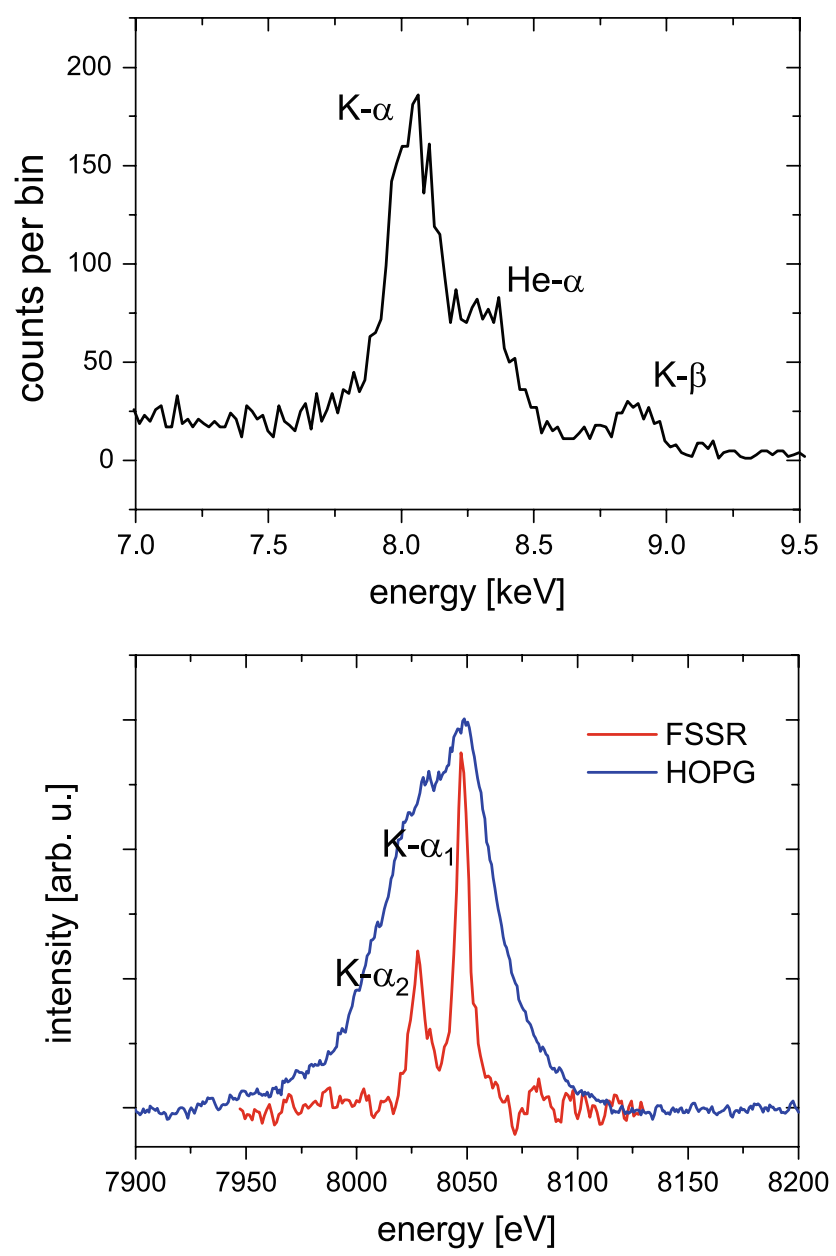

Fig. 10 Spectrum of the copper K-shell emission obtained with the single-photon counting camera (top). Emission from higher charge states up to He-like ions indicates strong heating of the target. High-resolution spectra of the K-alpha emission are obtained with the HOPG and mica (FSSR) crystal spectrometers (bottom)

for this class of experiments [27]. Besides strong K-alpha emission the spectra show $\mathrm{K}$-beta and $\mathrm{He}$-alpha radiation which can serve as a diagnostics of the target temperatures reached [28]. Advanced X-ray scattering techniques [29] require a higher spectral resolution to measure Doppler broadening [30] or to resolve plasmon resonances in the scattering spectrum [31]. Low Thomson scattering cross-section necessitates powerful sources and a high collection efficiency 
of the scattering spectrometer. In favor for that purpose, highly oriented pyrolytic graphite (HOPG) crystals provide an exceptionally high peak reflectivity [32]. At GSI we used a 70-mm large curved HOPG crystal in a van Hamos type spectrometer. Coupled with imaging-plate detectors, this constitutes a highly efficient spectrometer suitable for $\mathrm{X}$-ray scattering diagnostics. The resolution is limited to $E / \Delta E \sim 200$ due to depth broadening and aberrations due to the large mosaicity of the HOPG crystals. A much higher resolution can be provided by a focusing spectrometer that employs a spherically bent Mica crystal as focusing optical element. In one direction of the imaging plane, the resolving power of this spectrometer reaches 3000; and in the other direction, it provides in addition high-resolution onedimensional spatial imaging (see, e.g., [33]), allowing for the determination of the source size with a $10-\mu \mathrm{m}$ accuracy. The spectrum of hard X-ray bremsstrahlung was assessed using an 8-channel detector based on an imaging plate with lead filters of varying thickness up to $2 \mathrm{~mm}$. The response is simulated assuming a Boltzmann-type spectral distribution and using the filter transmission and image plate spectral sensitivity. We observe a strong dependence of the bremsstrahlung spectrum and consequently on the hot electron temperature on the focused intensity. While in some applications this background is detrimental, it is also a powerful backlighter for radiographing macroscopic dense high-Z targets.

Further experiments used the PHELIX laser system at highest intensities, around $5 \times 10^{19} \mathrm{~W} / \mathrm{cm}^{2}$. The intensity on target was measured using a novel nuclear pyrometry; a combination of selected isotopes is excited via $(\gamma, \mathrm{xn})$ reactions near the giant dipole resonance (GDR). Due to the energy-dependent cross-sections, the bremsstrahlung spectra and therefore the electron distribution and laser intensity could be confirmed. This diagnostics allows for measuring the real on-target intensity regardless of the influence of preplasma conditions.

The interaction between an ultra-intense laser pulse $(I>$ $10^{18} \mathrm{~W} / \mathrm{cm}^{2}$ ) and a thin target foil leads to an acceleration of protons up to kinetic energies of several tens of $\mathrm{MeV}$. In the short acceleration time of a few picoseconds, up to $10^{13}$ protons and ions are accelerated. Well-defined, smooth beams with transverse emittances up to hundred times smaller than in conventional accelerators are observed in experiments $[34,35]$. In the very first experiments, we already observed a very laminar proton beam of highest quality and up to $30 \mathrm{MeV}$ when focusing the PHELIX beam onto a $20 \mu \mathrm{m}$ gold foil. The protons were detected in stacked layers of GaFCromic radio-chromic film (RCF) where they cause a color change from transparent to dark blue according to their deposited energy. The protons were detected up to the 34th layer corresponding to an energy of $30 \mathrm{MeV}$ and the proton-energy spectrum was extracted from these images (see Fig. 11).

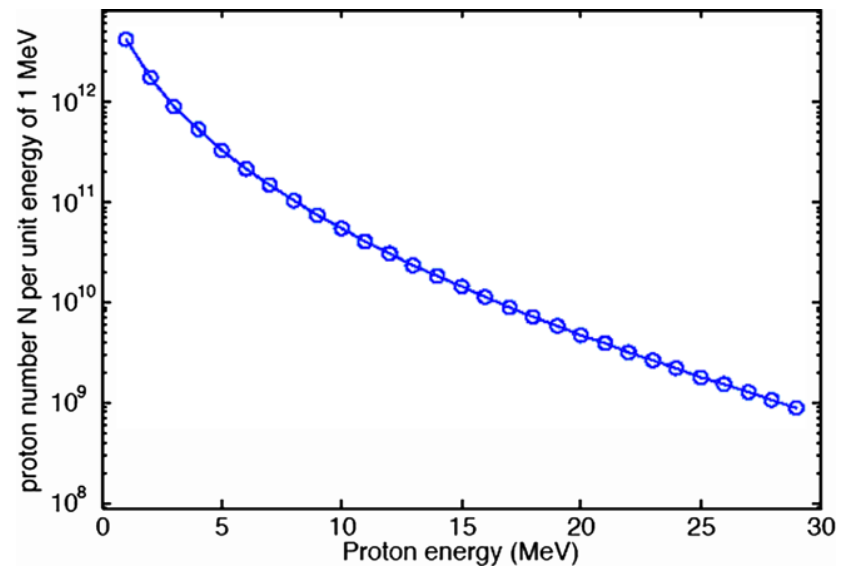

Fig. 11 PHELIX accelerated proton spectrum extracted from a stack of GAFCromic radio-chromic films

This remarkably good beam quality motivates the injection into conventional accelerators. However, such an application requires further investigations to reduce the halfopening angle of the beam, which currently is up to 40 degrees, and to minimize the energy spread. An external magnetic field as a collimation and energy filtering method decouples the acceleration process and the transport providing the opportunity of independent optimization of both processes. In the experiment described in Fig. 12, we used a pulsed high-field solenoid to collimate and focus the proton beam and further increase the proton flux density. Collimation or focusing is achieved by the Lorentz force generated by the particles when crossed into the radial and axial magnetic-field components of the solenoid. Since the solenoid focuses the beam in all transverse directions it can be used as a compact single device. Thus, higher transmission of protons through the solenoid can be achieved in comparison to the quadrupoles. The strong magnetic-flux densities of up to $15 \mathrm{~T}$ were generated by a pulsed-power device employing some of the capacitors normally used to energize the flash lamps of PHELIX. The solenoid was placed $17 \mathrm{~mm}$ behind the target to ensure that all protons enter the solenoid's aperture of $44 \mathrm{~mm}$. A magnetic-flux density of $8 \mathrm{~T}$ leads to a collimation of protons with a kinetic energy of $2.5 \mathrm{MeV}$. The measured particle numbers detected behind the solenoid at a magnetic-flux density of $8 \mathrm{~T}$ was compared to the initial particle numbers. Therefore, the detector was placed $240 \mathrm{~mm}$ behind the target and had a size of $(5 \times 5) \mathrm{cm}$ (see Fig. 12). As the simulation suggests, the $2.3 \mathrm{MeV}$ protons are slightly focused, so that the beam diameter behind the solenoid is smaller than the detector size.

Even though this has been just the beginning of a set of experimental beam-times dedicated to capture and transport laser accelerated proton beams at GSI, we already successfully could detect about $95 \%+5 /-19 \%$ of the initial particles at the chosen energy. 
Fig. 12 Collimating effect of a pulsed solenoid on ions accelerated by laser. The radio-chromic film (RCF) stack is located away from the target plane such that the amount of particle hitting it is not detectable when the solenoid is not pulsed (a). When the coil is energized, a clear proton beam signal (b) can be detected

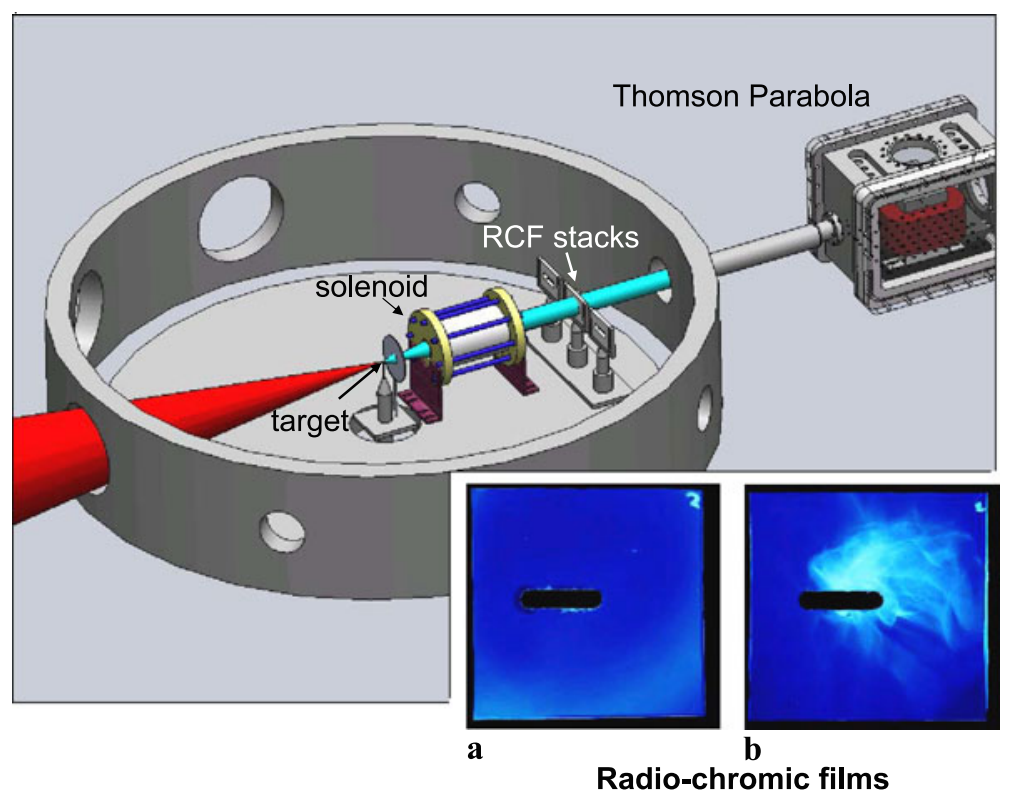

Radio-chromic films

\section{Conclusion and perspectives}

To summarize, PHELIX has demonstrated excellent performance in the high intensity operation mode as well as in the long pulse mode, in a way that fulfills the needs of the research program at GSI. PHELIX offers opportunities for international users involved in a wide range of experiments from the generation of high quality ion beams that can be transported through ion optical systems with high efficiency, to low-threshold X-ray laser emission at short wavelengths and incoherent hard X-ray emission.

Near-term upgrades of the facility include the improvement of the temporal contrast of the laser pulses, frequency doubling for long-pulse operation in order to avoid back reflections, the development of tight focusing capabilities at the UNILAC experimental hall for hohlraum heating, and finally the installation of a short pulse beamline of a somehow reduced power $(100 \mathrm{TW})$ at the accelerator, in order to couple laser-accelerated particles into conventional accelerator structures.

These projects as well as the research program at PHELIX will also benefit from the newly funded Helmholtz Institute Jena (HIJ), whose goal is to promote research at the interface of particle accelerators and high power lasers. The scope of the institutes includes challenging projects in basic research such as strong field QED and WDM, both strongly related to the realm of extreme states of matter. It also deals with the laser technology relevant to high power lasers in the context of the FAIR and XFEL projects and it offers the scientific environment necessary to keep the performance of PHELIX in phase with the ever-increasing experimental requirements.
Acknowledgements The successful completion of PHELIX and quality of operation depended on a number of very different valuable contributions. Basis for the whole project was the endowment of expensive large diameter laser components from the former Nova and Phebus systems by DOE and CEA. In this matter, our sincere thanks go to J. Caird, M. Campbell, R. McKnight, G. Logan, and M. Perry, and A. Bettinger, M. Decroisette and F. Kovacs. We also thank D. Habs (LMU München), F. Krausz (MPQ München), W. Sandner (MBI Berlin) and R. Sauerbrey (FZD Rossendorf) for their contributions. Important laser developments and access of European researchers was supported by the European International Infrastructure Initiative Laserlab Europe.

Open Access This article is distributed under the terms of the Creative Commons Attribution Noncommercial License which permits any noncommercial use, distribution, and reproduction in any medium, provided the original author(s) and source are credited.

\section{References}

1. M.D. Perry, D. Pennington, B.C. Stuart, G. Tietbohl, J.A. Britten, C. Brown, S. Herman, B. Golick, M. Kartz, J. Miller, H.T. Powell, M. Vergino, V. Yanovsky, Opt. Lett. 24, 160 (1999)

2. C.N. Danson, P.A. Brummitt, R.J. Clarke, J.L. Collier, B. Fell, A.J. Frackiewicz, S. Hancock, S. Hawkes, C. Hernandez-Gomez, P. Holligan, M.H.R. Hutchinson, A. Kidd, W.J. Lester, I.O. Musgrave, D. Neely, D.R. Neville, P.A. Norreys, D.A. Pepler, C.J. Reason, W. Shaikh, T.B. Winstone, R.W.W. Wyatt, B.E. Wyborn, IAEA J. Nucl. Fus. 44, S239 (2004)

3. E.W. Gaul, M. Martinez, J. Blakeney, M. Ringuette, D. Hammond, A. Jochmann, R. Escamilla, T. Borger, G. Dyer, T. Ditmire, in Conference on Lasers and Electro-Optics/International Quantum Electronics Conference, OSA Technical Digest (CD) (Optical Society of America, 2009), paper JWB2

4. E.I. Moses, R.L. McCrory, D.D. Meyerhofer, C.J. Keane, Opt. Photonics News 20, 42 (2009)

5. http://www.fair-center.org 
6. HEDgeHOB Collaboration, Technical report for the HEDgeHOB collaboration experiments, in FAIR Base-line Technical Report, ed. by H.H. Gutbrod (GSI, Darmstadt, 2006), or http://hedgehob.physik.tu-darmstadt.de

7. WDM Collaboration, Radiative properties of warm dense matter produced by intense heavy ion beams, in FAIR Baseline Technical Report, ed. by H.H. Gutbrod (GSI, Darmstadt, 2006), or http://www.gsi.de/forschung/phelix/Experiments/FAIR/ WDM/index e.html

8. R. Bock, S. Borneis, Ch. Bruske, J. Caird, D. Habs, D. Hoffmann, H. Kluge, Th. Kühl, D. Marx, P. Nickles, M. Perry, M. Roth, W. Sandner, W. Seelig, A. Tauschwitz, in Inertial Fusion Sciences and Applications 99, ed. by C. Labaune, W.J. Hogan, K.A. Tanaka (Elsevier, Paris, 2000), p. 703

9. D.H.H. Hoffmann, A. Blazevic, P. Ni, O. Rosmej, M. Roth, N. Tahir, A. Tauschwitz, S. Udrea, D. Varentsov, K. Weyrich, Y. Maron, Laser Part. Beams 23, 395 (2005)

10. Th. Stöhlker, H. Backe, H.F. Beyer, F. Bosch, A. BräuningDemian, S. Hagmann, D.C. Ionescu, K. Jungmann, H.-J. Kluge, C. Kozhuharov, Th. Kühl, D. Liesen, R. Mann, P.H. Mokler, W. Quint, Nucl. Instrum. Methods B 205, 156 (2003)

11. http://www.laserlab-europe.net/

12. G. Schaumann, M.S. Schollmeier, G. Rodrigez-Prieto, A. Blazevic, E. Brambrink, M. Geissel, S. Korostiy, P. Pirzadeh, M. Roth, F.B. Rosmej, A.Ya. Faenov, T.A. Pikuz, K. Tsigutkin, Y. Maron, N.A. Tahir, D.H.H. Hoffmann, Laser Part. Beams 23, 503 (2005)

13. D. Beck, K. Blaum, H. Brand, F. Herfurth, S. Schwarz, Nucl. Instrum. Methods 527, 567 (2004)

14. D. Strickland, G. Mourou, Opt. Commun. 56, 219 (1985)

15. V. Bagnoud, F. Salin, J. Opt. Soc. Am. B 16, 188 (1999)

16. C.P.J. Barty, G. Korn, F. Raksi, C. Rose-Petruck, J. Squier, A.-C. Tien, K.R. Wilson, V.V. Yakovlev, K. Yamakawa, Opt. Lett. 21, 219 (1996)

17. H.-M. Heuck, P. Neumayer, T. Kuehl, U. Wittrock, Appl. Phys. B 84, 421 (2006)

18. D. Javorková, V. Bagnoud, Opt. Express 15, 5439 (2007)

19. D. Zimmer, B. Zielbauer, V. Bagnoud, U. Eisenbarth, D. Javorkova, T. Kuehl, Opt. Express 16, 10398 (2008)

20. D.H.H. Hoffmann, K. Weyrich, H. Wahl, Th. Peter, J. Meyer-terVehn, J. Jacoby, R. Bimbot, D. Gardès, M.F. Rivet, M. Dumail, C. Fleurier, A. Sanba, C. Deutsch, G. Maynard, R. Noll, R. Haas, R. Arnold, S. Maurmann, Z. Phys. A 330, 339 (1988)

21. J. Jacoby, D.H.H. Hoffmann, W. Laux, R.W. Müller, H. Wahl, K. Weyrich, E. Boggasch, B. Heimrich, C. Stöckl, H. Wetzler, S. Miyamoto, Phys. Rev. Lett. 74, 1550 (1995)

22. A. Blažević, V. Bagnoud, S. Borneis, U. Eisenbarth, A. Frank, J. Fils, S. Götte, M. Günther, T. Hahn, T. Heßling, M. Kreutz, S. Kunzer, J. Menzel, T. Merz-Mantwill, E. Onkels, A. Pelka, D. Reemts, O. Rosmej, M. Roth, M. Schollmeier, D. Schumacher, T. Stöhlker, A. Tauschwitz, H. Wahl, K. Witte, in GSI Annual Report 2008, Plasma-Physics-02 (2009), p. 315
23. D. Zimmer, V. Bagnoud, B. Ecker, U. Eisenbarth, J. Habib, D. Hochhaus, D. Javorkova, S. Kazamias, T. Kuehl, D. Ros, D. Ursescu, B. Zielbauer, Springer Proc. Phys. 130, 91 (2009)

24. D. Zimmer, V. Bagnoud, B. Ecker, D. Hochhaus, T. Kuehl, D. Ros, B. Zielbauer, B. Aurand, in Ultrafast Optics Conference, Arcachon, France (2009), paper p.2.25

25. N.A. Tahir, I.V. Lomonosov, A. Shutov, V.E. Fortov, M. Geissel, A.R. Piriz, C. Deutsch, D.H.H. Hoffmann, Nucl. Instrum. Methods A 606, 128 (2009)

26. E. Brambrink, H.G. Wei, B. Barbrel, P. Audebert, A. BenuzziMounaix, T. Boehly, T. Endo, C. Gregory, T. Kimura, R. Kodama, N. Ozaki, H.-S. Park, M. Rabec le Gloahec, M. Koenig, Phys. Plasmas 16, 033101 (2009)

27. H.-S. Park, D.M. Chambers, H.-K. Chung, R.J. Clarke, R. Eagleton, E. Giraldez, T. Goldsack, R. Heathcote, N. Izumi, M.H. Key, J.A. King, J.A. Koch, O.L. Landen, A. Nikroo, P.K. Pate, D.F. Price, B.A. Remington, H.F. Robey, R.A. Snavely, D.A. Steinman, R.B. Stephens, C. Stoeckl, M. Storm, M. Tabak, W. Theobald, R.P.J. Town, J.E. Wickersham, B.B. Zhang, Phys. Plasmas 13, 056309 (2006)

28. W. Theobald, K. Akli, R. Clarke, J.A. Delettrez, R.R. Freeman, S. Glenzer, J. Green, G. Gregori, R. Heathcote, N. Izumi, J.A. King, J.A. Koch, J. Kuba, K. Lancaster, A.J. MacKinnon, M. Key, C. Mileham, J. Myatt, D. Neely, P.A. Norreys, H.-S. Park, J. Pasley, P. Patel, S.P. Regan, H. Sawada, R. Shepherd, R. Snavely, R.B. Stephens, C. Stoeckl, M. Storm, B. Zhang, T.C. Sangster, Phys. Plasmas 13, 043102 (2006)

29. S.H. Glenzer, R. Redmer, Rev. Mod. Phys. (2009, to be published)

30. S.H. Glenzer, G. Gregori, R.W. Lee, F.J. Rogers, S.W. Pollaine, O.L. Landen, Phys. Rev. Lett. 90, 175002 (2003)

31. S.H. Glenzer, O.L. Landen, P. Neumayer, R.W. Lee, K. Widmann, S.W. Pollaine, R.J. Wallace, G. Gregori, A. Höll, T. Bornath, R. Thiele, V. Schwarz, W.-D. Kraeft, R. Redmer, Phys. Rev. Lett. 98, 065002 (2007)

32. M.K. Urry, G. Gregori, O.L. Landen, A. Pak, S.H. Glenzer, J. Quant. Spectrosc. Radiat. Transf. 99, 636 (2006)

33. A.Ya. Faenov, S.A. Pikuz, A.I. Erko, B.A. Bryunetkin, V.M. Dyakin, G.V. Ivanenkov, A.R. Mingaleev, T.A. Pikuz, V.M. Romanova, T.A. Shelkovenko, Phys. Scr. 50, 333 (1994)

34. T.E. Cowan, J. Fuchs, H. Ruhl, A. Kemp, P. Audebert, M. Roth, R. Stephens, I. Barton, A. Blazevic, E. Brambrink, J. Cobble, J. Fernández, J.-C. Gauthier, M. Geissel, M. Hegelich, J. Kaael, S. Karsch, G.P. Le Sage, S. Letzring, M. Manclossi, S. Meyroneinc, A. Newkirk, H. Pépin, N. Renard-LeGalloudec, Phys. Rev. Lett. 92, 204801 (2004)

35. F. Nürnberg, M. Schollmeier, E. Brambrink, A. Blažević, D.C. Carroll, K. Flippo, D.C. Gautier, M. Geißel, K. Harres, B.M. Hegelich, O. Lundh, K. Markey, P. McKenna, D. Neely, J. Schreiber, M. Roth, Rev. Sci. Instrum. 80, 033301 (2009) 